\title{
Recent patents in optogenetics and optochemistry
}

\section{Patent number}

Description

US $9,676,836$

thods and compositions to identify and characterize new channelrhodopsins derived from algae and several of which are red-shifted. The rhodopsin domain of these red-shifted channelrhodopsins can be cloned and expressed in mammalian systems and used in optogenetic applications and as therapeutic agents. Also, methods and compositions for use in red-shifting the absorbance maxima of channelrhodopsins in order to improve their utility for use in vivo.

US 9,655,594 Methods and compositions for gelatin-based tissue-mimicking opto-acoustic phantom that accurately replicates opto-acoustic properties of biological tissue and permits matching of each optical and each acoustic property of specific tissues independently so that by changing one property the other property is not altered. Such phantoms can match tissue properties in the specific range of system parameters required for evaluation of hardware and software performance, calibration, validation or personnel training of optical, optoacoustic, ultrasonic or combined systems used for imaging, sensing or monitoring of tissue morphology and molecular composition.

US $9,615,789$ Systems and methods involving the use of MRI and optogenetic neural stimulation, including modifying a target neural cell population in a first region of a brain to express light-responsive molecules. Using a light pulse, the light-responsive molecules in the target neural cell population are stimulated. Multiple regions of the brain are scanned via MRI. The scans allow for observation of a neural reaction in response to the stimulation in at least one of the multiple regions of the brain.

US 9,207,405 A hybrid rotary joint providing both optical and fluid channels for applications such as optogenetic research. The joint has a stationary component and a rotating component. The optical channel and fluid channel permit simultaneous and rotation-insensitive passage of light and fluid from the stationary to the rotating side of the hybrid rotary joint. The input to the optical channel is provided via an input optical fiber and the input to the fluid channel is fluid from a fluid source. The outputs are an optical fiber output and a fluid channel output. As light passes from the stationary side to the rotating side of the hybrid rotary joint, it is deflected off a beam deflector having an angular orientation that is passively aligned and made independent of the angular orientation of the rotating component via the interaction between magnets attached to each of the beam deflector and the stationary component.

US 9,091,660 A device for measuring at least one parameter of an arterial blood sample, including a flow sensor having standardized connecting elements, which are connected to the inlet and the outlet side of a measuring cell of the flow sensor, the measuring cell having at least one optochemical sensor element which can be brought in contact with the blood sample. A connector is removably placed on the flow sensor and includes at least one light source for exciting the optochemical sensor element and at least one photodetector for receiving the measurement radiation of the optochemical sensor element. An electronic module has an electric connecting line to the connector and contacts at least one light source and at least one photodetector, the flow sensor being connectible to the standardized connection of an arterial catheter via the connecting element on the inlet side and to the standardized connection of an arterial infusion set via the connection on the outlet side.

US 9,046,659 A dual optical path rotary joint provides a low friction coupling of optical fiber connections for optogenetic research and other applications. Two optical paths are provided through the rotary joint which are rotation-insensitive. The ends of the rotary joint rotate with respect to a common housing, which may be provided with ball-bearings or other suitable low-friction rotational mounts in which a first rotating component and a second rotating component are secured. The first and second rotating components are not mechanically linked in rotation, and their rotation is only synchronized by magnets disposed on the first rotating component and the second rotating component. A pair of optical fibers is coupled to the first rotating component and another pair of optical fibers is coupled to the second rotating component. A pair of isolated optical paths links the pairs of fibers, which are directed between the first and second rotating components by reflection.

US 8,868,351 Systems and methods for estimating a volume of tissue directly influenced by optogenetic stimulation. The systems and methods can also enable a user to quantitatively predict the spread of stimulation (e.g., action potential) resulting from optical stimulation in a patient's tissue.

\section{Assignee}

Board of Regents

of the University

of Texas System

(Austin, TX, USA)

Seno Medical

Instruments (San

Antonio, TX, USA)

Tsyboulski DA, Miller TG

Board of Trustees

of Stanford

Deisseroth K,

4/11/2017

University (Stanford,

CA, USA)

Optomak

Canada)
Date

6/13/2017

udich $J L$ Govorunova EG Sineshchekov

$\mathrm{OA}$

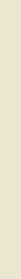

Smart Medical
Solutions

(Graz, Austria)
Koehler H

7/28/2015

Optomak (Quebec, Doric S Canada)
The Cleveland Clinic Foundatio

(Cleveland, $\mathrm{OH}$, USA)
McIntyre CC, Foutz TJ 